\title{
ABORDAGEM CIRÚRGICA IMEDIATA DE FRATURA DE SEIO FRONTAL: RELATO DE CASO
}

\author{
IMMEDIATE SURGICAL APPROACH OF FRONTAL SINUS FRACTURE: A CASE REPORT
}

\author{
Cristóvão Marcondes de Castro RODRIGUES ${ }^{1}$; Izabella SOL'; Vinicius Lima de ALMEIDA; Danyella Carolyna Soares dos REIS²; \\ Claudia Jordão SILVA3; Lívia Bonjardim LIMA3; Luiz Fernando Barbosa de PAULO \\ 1 - Residente de Cirurgia e Traumatologia Bucomaxilofacial, Hospital de Clínicas da Universidade Federal de Uberlândia (HC-UFU), Uberlândia, \\ Minas Gerais, Brasil. \\ 2 - Mestranda em Clínica Odontológica pela Universidade Federal de Uberlândia. Cirurgiã-dentista formada pela Faculdade de Odontologia da \\ Universidade Federal de Uberlândia (HC-UFU). Uberlândia, Minas Gerais, Brasil. \\ 3 - Preceptor da Residência de Cirurgia e Traumatologia Bucomaxilofacial, Hospital de Clínicas da Universidade Federal de Uberlândia (HC-UFU), \\ Uberlândia, Minas Gerais, Brasil.
}

\section{RESUMO}

Objetivo: O seio frontal é uma cavidade óssea pneumática, localizada entre o esplenocrânio e o neurocrânio, contidos entre a fossa craniana anterior e a região naso-órbito-etmoidal. As fraturas do seio frontal são originadas a partir de acidentes de grande intensidade. Traumas na região pode afetar a lâmina anterior e/ ou posterior, com ou sem envolvimento do ducto nasofrontal. $\mathrm{O}$ tratamento das fraturas do seio frontal de forma adequada apresenta grande significado clínico, pois as sequelas geradas podem trazer transtornos funcionais e estéticos importantes, envolvendo relação multiprofissional entre as equipes de neurocirurgia e cirurgia buco-maxilo-facial. O objetivo deste artigo é relatar um caso de intervenção cirúrgica imediata para remoção de corpo estranho na região seio frontal e redução e fixação da fratura seio frontal após acidente automobilístico em paciente jovem do gênero masculino. Material e Método: Sob indução de anestesia geral, foi realizado redução e fixação da fratura do osso frontal por meio de mini-placas de titânio. Resultados: Paciente evoluiu de maneira satisfatória, com restabelecimento do contorno estético aceitável do osso frontal, sem qualquer cosmético e funcional. Conclusão: Mesmo com as incongruências na literatura atual, em relação ao momento de abordagem de fraturas relacionadas ao seio frontal, o manejo mais precoce possível se mostra uma vertente com resultados satisfatórios, e com menores índices de complicações, devendo ser considerado sempre como uma possibilidade.

PALAVRAS-CHAVE: Seio frontal; Osso frontal; Traumatismos faciais; Face.

\section{INTRODUÇÃO}

A fratura do osso frontal corresponde entre 3-5\% das fraturas de face ${ }^{1}$. Os seios frontais, quando desenvolvidos, se localizam entre as lâminas interna e externa do osso frontal, e ambas as paredes podem ser muito finas ${ }^{2}$. As fraturas de seio frontal podem acometer anatomicamente as paredes anterior e/ou posterior, com ou sem envolvimento do ducto nasofrontal ${ }^{3}$.

É relatado na literatura que a idade média dos pacientes com fraturas de frontal é de 29,1 anos e que cerca de 92,3\% dos pacientes pertencem ao gênero masculino. As causas mais comuns de fratura são acidentes automobilísticos e agressão física ${ }^{4}$.

Nos casos de fraturas, podem haver várias complicações para o paciente, entre elas a depressão frontal, epistaxe, obstrução nasal e possíveis alterações neurológicas ${ }^{5}$. Seu tratamento é cirúrgico (salvo em casos de fraturas sem deslocamento ou nas que o paciente não quer) e exige o uso de placas/parafusos de titânio e/ou tela de titânio e/ou biomateriais para preenchimento em caso de perda óssea avançada ${ }^{5}$.

Por se tratar, na grande maioria dos casos, de pacientes politraumatizados, a interação multiprofissional entre as equipes de neurocirurgia e buco-maxilo-facial é primordial para o melhor planejamento e execução cirúrgica ${ }^{6}$.

Objetivo deste artigo foi fazer relato de caso de uma intervenção cirúrgica imediata para tratamento de uma fratura de seio frontal associada à presença de uma transposição de corpo estranho em um paciente jovem do gênero masculino, após acidente automobilístico.

\section{RELATO DE CASO}

Paciente de 26 anos, leucoderma, gênero masculino foi encaminhado para o Hospital de Clínicas da Universidade Federal de Uberlândia após acidente automobilístico. A equipe da cirurgia geral realizou a avaliação primária, e o paciente encontrava-se lúcido e consciente sem apresentar instabilidades sistêmicas e neurológicas. Foram solicitados exames de imagem tomografia computadorizada de crânio e face, e as equipes da neurocirurgia e bucomaxilofacial foram acionadas para avaliação.

No exame clínico neurológico e na avaliação da tomografia de crânio do paciente não foi observado comprometimento 
neurológico que necessitasse de intervenção por parte da equipe de neurocirurgia. A equipe da bucomaxilofacial, após avaliação clínica inicial, constatou a presença de laceração extensa na região frontal, com comprometimento do seio frontal e a presença de um corpo estranho alojado no ferimento. Na tomografia de face, notou-se no corte axial (Figura 1A), no corte coronal (Figura 1B) e na reconstrução 3D (Figura 1C) fratura da parede anterior do seio frontal, em três segmentos, e no corte sagital (Figura 1D) nota-se ausência de comprometimento da parede posterior. Durante avaliação primária o paciente não referiu uso de substâncias ilícitas e negou alterações sistêmicas referindo apenas alergia a dipirona, logo o mesmo foi submetido à intervenção cirúrgica imediata para remoção do corpo estranho e redução e fixação da fratura seio frontal, sob anestesia geral.

Posteriormente à indução anestésica, foi realizado limpeza da laceração (Figura 2A) com clorexidina 2\% (Riohex®) e lavagem copiosa com soro fisiológico, posteriormente foi infiltrado anestésico bupivacaína $0,5 \%$ com vasoconstritor 1:100.000 de noradrenalina Neocaina ${ }^{\circledR}$ em pontos estratégicos da laceração. O corpo estranho foi removido (Figura 3), curetagem e irrigação copiosa do leito cirúrgico com soro fisiológico foram realizadas e verificado a manutenção da patência do ducto nasofrontal. A fratura da parede anterior do seio apresentou perda de dois fragmentos, sendo possível a redução e fixação apenas no maior fragmento, sendo utilizadas miniplacas de fixação do sistema 1.5 mm (Neoortho $\left.{ }^{\circledR}\right)$ (Figura 2B), após fixação da fratura, uma irrigação abundante e criteriosa com soro fisiológico foi realizada, e sutura da laceração foi realizada em planos com fio vicryl 3-0 (Procare ${ }^{\circledR)}$ e para sutura em pele foi utilizado nylon 5-0 (Procareß) (Figura 2C). Um curativo compressivo foi feito e mantido por três dias. Paciente seguiu de alta hospitalar com prescrição domiciliar de levofloxacino $500 \mathrm{mg} 2 \mathrm{x}$ ao dia por sete dias, dexametasona $4 \mathrm{mg} 1 \mathrm{x}$ ao dia por três dias e paracetamol $750 \mathrm{mg}$ $3 \mathrm{x}$ dia por três dias.

No retorno ambulatorial de sete dias, paciente não apresentou queixas, a ferida operatória apresentava bom aspecto, e foi realizada a remoção das suturas.

Após sessenta dias da intervenção cirúrgica o paciente não apresentava queixas, nenhum tipo de sinal flogístico. Foi realizado uma tomografia computadorizada para avaliação, sendo observada nos cortes axial (Figura 4A), coronal (Figura 4B), reconstrução 3D (Figura 4C) e sagital (Figura 4D) uma satisfatória redução e restabelecimento no contorno do osso frontal reconstituindo contorno estético e o mesmo seguiu de alta ambulatorial.

\section{DISCUSSÃO}

As fraturas do osso frontal são normalmente associadas a um trauma de alta energia. As etiologias mais frequentes são acidente automobilístico seguido de agressão física, queda acidental, acidentes esportivos, acidente ciclístico, atropelamento e acidentes com animais ${ }^{7}$. No caso descrito o fator etiológico corrobora com os achados na literatura, por se tratar de um acidente de automobilístico de alta energia.

Para correto diagnóstico, os exames clínicos associados aos exames por imagem são fundamentais, sendo a tomografia computadorizada um exame essencial e considerado padrão ouro para o diagnóstico de fratura seio frontal visto que permite avaliação do grau de cominuição, comprometimento ducto nasofrontal, se existe o acometimento da parede posterior do seio e assim permitindo um melhor diagnóstico e adoção do plano tratamento ${ }^{6,8}$. As tomadas radiográficas de crânio, lateral de crânio, Towne, Waters, são meios utilizados para o

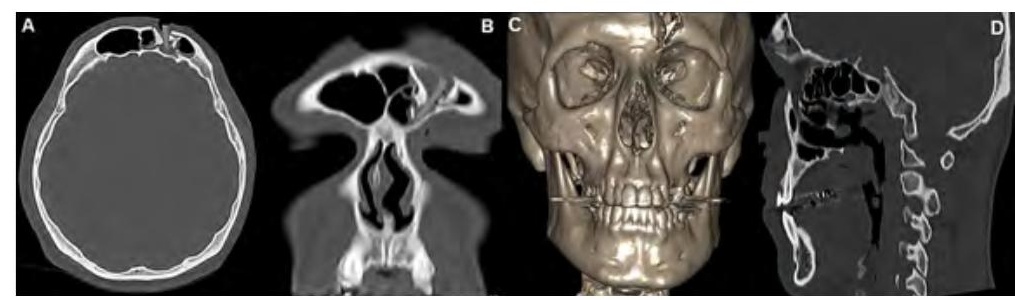

Figura 1 - Corte axial de tomografia computadorizada apresentando solução de descontinuidade óssea parede anterior de seio frontal (A), Corte coronal de tomografia computadorizada apresentando velamento seio frontal, artefato intrínseco (B), Reconstrução 3D de tomografia computadorizada evidenciando fratura osso frontal (C), Corte sagital de tomografia computadorizada apresentando solução de descontinuidade óssea parede anterior de seio frontal e velamento seio frontal (D).

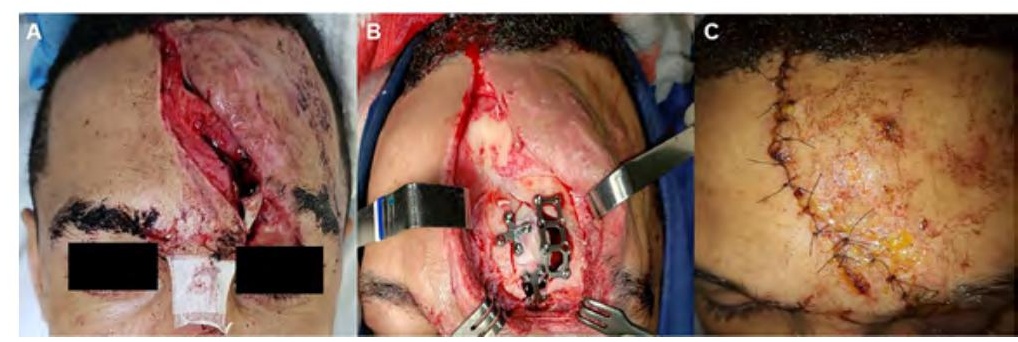

Figura 2 - Laceração extensa região frontal (A), Redução e fixação da fratura osso frontal com mini-placas do sistema 2.0 (B), Aspecto pós-operatório de sete dias após abordagem cirúrgica $(\mathrm{C})$.

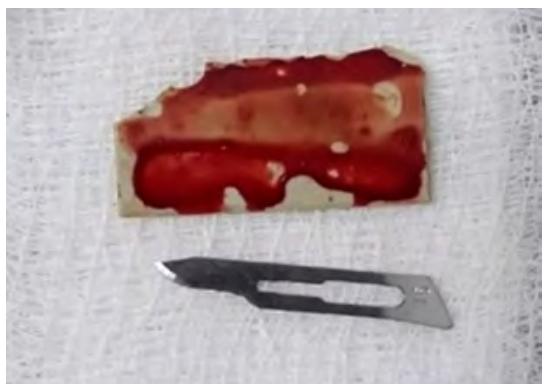

Figura 3 - Material plástico (corpo estranho) removido do interior do seio frontal.

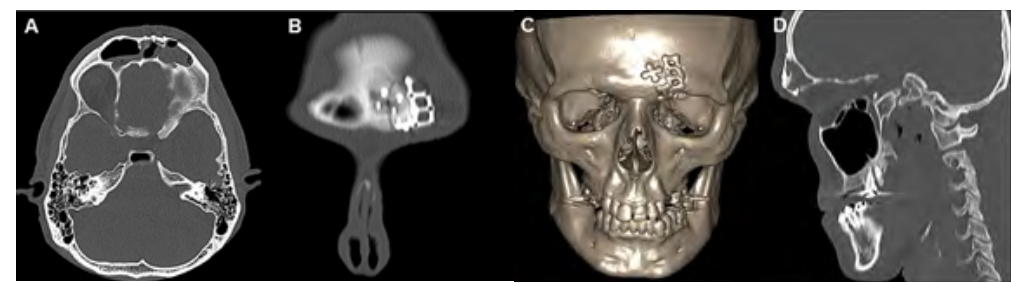

Figura 4 - Corte axial de tomografia computadorizada apresentando a reconstituição anatômica da parede anterior de seio frontal (A), Corte coronal de tomografia computadorizada apresentando posicionamento do material de osteossíntese (B), Reconstrução 3D de tomografia computadorizada evidenciando reestruturação anatômico do osso frontal (C), Corte sagital de tomografia computadorizada apresentando reconstituição óssea parede anterior de seio frontal (D). 
diagnóstico fratura do osso frontal, quando exames tomográficos não se apresentam disponiveis ${ }^{8}$. Com as imagens tomográficas realizadas na admissão do paciente, foi observada ausência de comprometimento da tábua posterior do seio frontal e do ducto nasofrontal e assim permitindo mais segurança na intervenção cirúrgica imediata, devido ao risco neurológico mínimo.

O tratamento das fraturas do seio frontal constitui uma fonte de controvérsias, apesar do progresso obtido com os atuais modelos de fixação rígida e evolução das técnicas operatórias ${ }^{9}$. Os principais materiais usados no tratamento das fraturas craniofaciais são confeccionados em titânio devido sua excelente biocompatibilidade e fácil manuseio de suas placas e telas ${ }^{4,6,8}$. Defeitos ósseos pequenos podem ser solucionados apenas com placas e telas enquanto os maiores são associados a enxertos ${ }^{8}$. Fraturas da parede anterior do seio frontal sem envolvimento da parede posterior ou de injuria do ducto nasofrontal podem ser reconstruídas e, fixadas rigidamente com miniplacas e parafusos $^{6}$. Enxertos ósseos podem, também, ser utilizados quando houver cominuição excessiva ou perda óssea, já nas fraturas de parede posterior, em certos casos se faz necessário à reposição ou remoção (cranialização) ${ }^{10}$. A cranialização deve ser especialmente realizada quando houver cominuição da parede posterior, lesão da dura-máter e drenagem de líquor ${ }^{9,10,11}$. No presente caso por se tratar de trauma cominuto, mas não extenso, e sem comprometimento da parede posterior do seio frontal, foi possível aproveitar parte dos fragmentos ósseos e realizar fixação com miniplacas de titânio.

Outro ponto controverso na literatura é a via de abordagem da fratura do seio frontal; nas fraturas simples envolvendo principalmente a parede anterior, prefere-se a incisão abaixo da sobrancelha à incisão bicoronal, ou em casos em que existam lacerações ou lesões corto contusas que permitam o acesso à fratura também é uma escolha a ser considerada ${ }^{12}$. Assim como no presente caso, a presença de laceração região frontal permitiu acesso direto a fratura, assim não sendo necessária a utilização de nenhum tipo de acesso cirúrgico.

Observações de diversos autores mostram que o momento de abordagem do paciente com fratura do seio frontal é ainda controverso, Rohrich e Hollier ${ }^{13}$ (1992) mostraram que a cirurgia, logo após o trauma, diminui a chance de morbidade e apresentam melhores prognósticos. Deste modo a equipe responsável decidiu por realizar a intervenção cirúrgica de maneira imediata, diminuindo risco de complicações e morbidade do ato cirúrgico.

\section{CONCLUSÃO}

As fraturas de seio frontal têm sido cada vez mais recorrentes devido aos acidentes de alta energia, e desta forma tornando tal condição mais presente no cotidiano do cirurgião buco-maxilo-facial.

A decisão do melhor momento para o tratamento e a técnica a ser empregada dependerá da gravidade e extensão da lesão e de uma completa avaliação clínica do paciente e do trauma.

No caso descrito, abordagem imediata foi possível, devido ao quadro neurológico e hemodinâmico estável do paciente. A presença da laceração permitiu intervenção direta da fratura, sem a necessidade de realizar acesso cirúrgico convencional. $\mathrm{O}$ resultado final foi muito satisfatório na perspectiva do paciente e da equipe responsável pela execução da cirurgia.
Os objetivos do tratamento cirúrgico da fratura do seio frontal são a prevenção de infecção, o isolamento do conteúdo intracraniano e restauração da função e estética.

\section{REFERÊNCIAS}

01. Lessa ES, Cruz RL, Costa MJM, Magalhães GE, Braune AS. Fraturas do seio frontal: conduta em relação ao ducto nasofrontal. Rev Bras Cir Plást. 2010; 25(3): 19.

02. Mensink, G; Zweers, A; Van Merkesteyn, JPR. Endoscopically assisted reduction of anterior table frontal sinus fractures. J Craniomaxillofac Surg. 2009; 37(4): 225-228.

03. Dalla DT , Burtscher D , Kloss AB , Rasse M, Kloss F. Manejo de fraturas do seio frontal - decisão do tratamento com base na extensão da luxação métrica. J Craniomaxillofac Surg. 2014; 42(7): 1515-9.

04. Gabrielli MF, Gabrielli MA, Hochuli-Vieira E, Pereira-Filho VA. Reconstrução imediata de fraturas do seio frontal: revisão de 26 casos. J Oral Maxillofac Surg. 2004; 62(5): 582-6.

05. Tedaldi M, Ramieri V, Foresta E, Cascone P, Iannetti G. Experience in the management of frontal sinus fractures. J Craniofac Surg. 2010; 21(1): 208-210.

06. Schultz K, Braun TL, Truong TA. Fraturas do seio frontal. Semin Plast Surg. 2017; 31(2): 80-84.

07. Montovani JC, Nogueira EA, Ferreira FD, Neto ACL, Nakajima V. Cirurgia das fraturas do seio frontal: estudo epidemiológico e análise de técnicas. Rev Bras Otorrinolaringol. 2006; 72(2): 204-209.

08. Kamoshima Y, Terasaka S, Nakamaru Y, Takagi D, Fukuda S, Houkin K. Giant frontal mucocele occurring 32 years after frontal bone fracture: a case report. Case Rep Neurol. 2012; 4(4): 34-37.

09. Silva JJ, Rocha Neto A, Pereira AM, Correia V, Lira AA. Fratura tardia de seio frontal: relato de caso clínico. Rev Cir Traumatol Bucomaxilo-fac. 2005; 5(3): 51-56.

10. Kuttenberger JJ, Hardt N. Long-term results following reconstruction of craniofacial defects with titanium micromesh systems. J Craniomaxillofac Surg. 2001; 29(2): 75-78.

11. Langford RJ, Frame JW. Tissue changes adjacente to titanium pates in patients. J Craniomaxillof Surg. 2002; 30(2): 103-107.

12. Metzinger SE, Metzinger RC. Complications of Frontal Sinus Fractures. Craniomaxillofac Trauma Reconstr. 2009; 2(1): 27-34.

13. Rohrich RJ, Hollier LH. Management of frontal sinus fractures. Changing concepts. Clin Plast Surg. 1992; 19(1): 219-32.

\section{ABSTRACT}

Objective: The frontal sinus is a pneumatic bone cavity located between the splenocranium and the neurocranium, contained between the anterior cranial fossa and the naso-orbital-ethmoid region. Frontal sinus fractures originate from accidents of high intensity. Trauma to the region can affect the anterior and / or posterior lamina, with or without nasofrontal duct involvement. Proper treatment of frontal sinus fractures has great clinical significance, as the sequelae generated can bring important functional and aesthetic disorders, involving a multi-professional relationship between neurosurgery and facial buccomaxillary surgery teams. The aim of this paper is to report a case of immediate surgical intervention for removal of the frontal sinus foreign body and reduction and fixation of the frontal sinus 
fracture after a car accident in a young male patient. Material and Method: Under induction of general anesthesia, a reduction and fixation of the frontal bone fracture was performed using titanium mini-plates. Results: Patient evolved satisfactorily, with restoration of the acceptable aesthetic contour of the frontal bone, without any cosmetic and functional. Conclusion: Even with the incongruencies in the current literature, in relation to the moment of approaching fractures related to the frontal sinus, the earliest possible management is shown to be an aspect with satisfactory results, and with lower rates of complications, and should always be considered as a possibility.

KEYWORDS: Frontal sinus; Frontal bone; Facial injuries; Face.

\section{AUTOR PARA CORRESPONDÊNCIA}

Cristóvão Marcondes de Castro Rodrigues

Departamento de Cirurgia e Traumatologia

Bucomaxilofacial e Implantodontia

Av. Pará, 1748 - Umuarama, Uberlândia - MG, 38405-320

Bloco 4T Uberlândia Minas Gerais-MG.

Telefone: (34) 99906-2400

E-mail: cristovao-marcondes@hotmail.com 